\title{
Some criteria for boundedness and compactness of the Hardy operator with some special kernels
}

\author{
Alois Kufner, Komil Kuliev and Ryskul Oinarov*
}

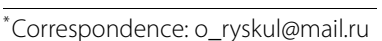
Astana, Republic of Kazakhstan

\begin{abstract}
We present necessary and sufficient conditions for boundedness and compactness of Hardy operator (1.4) with kernel (1.3) for $1<p \leq q<\infty$.

MSC: Primary 26D10; 26D15; secondary 47B07; 47B34; 47B38

Keywords: inequalities; Hardy-type inequalities; weights; Hardy operator; compact operator
\end{abstract}

\section{Introduction}

First, let us recall that the weighted Lebesgue space $L^{r}(w)$, with $r \geq 1$ and $w$ a weight function on $(0, \infty)$, is defined as a set of all functions $f=f(x)$ such that

$$
\int_{0}^{\infty}|f(x)|^{r} w(x) \mathrm{d} x<\infty
$$

We will investigate the Hardy-type inequality

$$
\left(\int_{0}^{\infty}\left|\int_{0}^{x} k(x, t) f(t) \mathrm{d} t\right|^{q} u(x) \mathrm{d} x\right)^{\frac{1}{q}} \leq C\left(\int_{0}^{\infty}|f(x)|^{p} v(x) \mathrm{d} x\right)^{\frac{1}{p}}
$$

with $1<p, q<\infty$ and $u, v$ weight functions on $(0, \infty)$.

This inequality was investigated by many authors. For $k(x, t) \equiv 1$, we obtain the 'classical' Hardy inequality

$$
\left(\int_{0}^{\infty}\left|\int_{0}^{x} f(t) \mathrm{d} t\right|^{q} u(x) \mathrm{d} x\right)^{\frac{1}{q}} \leq C\left(\int_{0}^{\infty}|f(x)|^{p} v(x) \mathrm{d} x\right)^{\frac{1}{p}}
$$

the case $k(x, t)=a(x) b(t)$ can be easily reduced to the classical case with modified weights $U(x)=|a(x)|^{q} u(x)$ and $V(x)=|b(x)|^{-p} v(x)$ instead of $u$ and $v$, respectively. Stepanov [1] has investigated 'convolutionary' kernels like $(x-t)^{\alpha}$, and probably the most general approach is connected with the name of Oinarov who investigated positive kernels $k$ such that

$$
k(x, t) \approx k(x, z)+k(z, t), \quad t<z<x
$$

@ 2013 Kufner et al.; licensee Springer. This is an Open Access article distributed under the terms of the Creative Commons Attribution License (http://creativecommons.org/licenses/by/2.0), which permits unrestricted use, distribution, and reproduction in any medium, provided the original work is properly cited. 
(see [2]) and also several more general kernels (see [3, 4]). Also, inequalities with modified or generalized kernels have been investigated; let us mention the recent book [5] where multiple Hardy-type inequalities with the so-called product kernels are considered. See also [6] and [7] for further details.

Here we consider kernels $k(x, t)$ of the type

$$
k(x, t)=\sum_{i=1}^{m} a_{i}(x) b_{i}(t)
$$

and we want to find conditions on the weight functions $u, v$ and on the functions $a_{i}, b_{i}$, for which the integral operator

$$
(k f)(x):=\int_{0}^{x} k(x, t) f(t) \mathrm{d} t
$$

maps the space $L^{p}(v)$ continuously into $L^{q}(u)$.

Remark 1.1 The case of kernel (1.3) with special functions $b_{i}(t)=t^{i-1}$ was investigated by Rychkov [8] for $p=q=2$. Such kernels appear for general $p, q$ by the investigation of higher-order Hardy inequalities; see [9].

Now, let us denote, for given $a_{i}, b_{i}, u$ and $v$,

$$
A_{i}(x)=a_{i}(x) u^{1 / q}(x), \quad B_{i}(t)=b_{i}(t) v^{-1 / p}(t) .
$$

Then we can rewrite inequality (1.1) [for functions $f$ ] as the unweighted inequality

$$
\left(\int_{0}^{\infty}\left|\int_{0}^{x} K(x, t) g(t) \mathrm{d} t\right|^{q} \mathrm{~d} x\right)^{\frac{1}{q}} \leq C\left(\int_{0}^{\infty}|g(x)|^{p} \mathrm{~d} x\right)^{\frac{1}{p}}
$$

[for functions $\left.g(x)=f(x) v^{1 / p}(x)\right]$ with

$$
K(x, t)=\sum_{i=1}^{m} A_{i}(x) B_{i}(t)
$$

Remark 1.2 If $m=1$, we have $K(x, t)=A(x) B(t)$, and we can rewrite (1.6) as

$$
\left(\int_{0}^{\infty}\left|\int_{0}^{x} B(t) g(t) \mathrm{d} t\right|^{q}|A(x)|^{q} \mathrm{~d} x\right)^{\frac{1}{q}} \leq C\left(\int_{0}^{\infty}|g(x)|^{p} \mathrm{~d} x\right)^{\frac{1}{p}},
$$

which is in fact the classical (weighted) Hardy inequality

$$
\left(\int_{0}^{\infty}\left|\int_{0}^{x} h(t) \mathrm{d} t\right|^{q}|A(x)|^{q} \mathrm{~d} x\right)^{\frac{1}{q}} \leq C\left(\int_{0}^{\infty}|h(x)|^{p}|B(x)|^{-p} \mathrm{~d} x\right)^{\frac{1}{p}}
$$

[for the function $h(x)=g(x) B(x)$ ] with the weight functions $|A(x)|^{q}$ and $|B(x)|^{-p}$. 
It is well known (see, e.g., [6] or [7]) that for the case $1<p \leq q<\infty$, inequality (1.8) holds for all functions $h \geq 0$ if and only if the so-called Muckenhoupt-Bradley condition

$$
\sup _{z>0} A_{M}(z)<\infty
$$

is satisfied, where

$$
\begin{aligned}
A_{M}(z) & =\left(\int_{z}^{\infty}|A(x)|^{q} \mathrm{~d} x\right)^{1 / q}\left(\int_{0}^{z}\left[|B(x)|^{-p}\right]^{1-p^{\prime}} \mathrm{d} x\right)^{1 / p^{\prime}} \\
& =\left(\int_{z}^{\infty}|A(x)|^{q} \mathrm{~d} x\right)^{1 / q}\left(\int_{0}^{z}|B(x)|^{p^{\prime}} \mathrm{d} x\right)^{1 / p^{\prime}}
\end{aligned}
$$

with $p^{\prime}=p /(p-1)$.

It follows from (1.9) that we need the integrability of $|A(x)|^{q}$ on $(z, \infty)$ and of $|B(x)|^{p^{\prime}}$ on $(0, z)$ for all $z>0$, i.e.,

$$
A \in L^{q}(z, \infty) \text { and } B \in L^{p^{\prime}}(0, z)
$$

[where we denote by $L^{r}(\alpha, \beta)$ the classical Lebesgue spaces of functions defined on $(\alpha, \beta)$ ].

In accordance with this remark, we suppose throughout the paper that the functions $A_{i}$, $B_{i}$ from (1.7) satisfy

$$
A_{i} \in L^{q}(z, \infty) \quad \text { and } \quad B_{i} \in L^{p^{\prime}}(0, z)
$$

for all $z>0$ and $i=1,2, \ldots, m$.

\section{Sufficient conditions}

For $p>1$ and $u, v$ weight functions on $(0, \infty)$, let us define

$$
A_{M}(x ; u, v)=A_{M}(x):=\left(\int_{x}^{\infty} u(t) \mathrm{d} t\right)^{\frac{1}{q}}\left(\int_{0}^{x} v^{1-p^{\prime}}(t) \mathrm{d} t\right)^{\frac{1}{p^{\prime}}}
$$

Remark 2.1 Let us recall that the condition

$$
\sup _{x>0} A_{M}(x)<\infty
$$

is necessary and sufficient for the validity of the classical Hardy inequality (1.2) for $p \leq q$.

It is easy to find a sufficient condition for (1.1) to hold, if we consider the 'partial' operators

$$
\left(k_{i} f\right)(x):=\int_{0}^{x} a_{i}(x) b_{i}(t) f(t) \mathrm{d} t
$$

as operators from $L^{p}(v)$ into $L^{q}(u)$. 
Theorem 2.2 Let $1<p \leq q<\infty$. For $A_{M}(x)$ defined by (2.1), denote

$$
A_{M, i}(x):=A_{M}\left(x ; u\left|a_{i}\right|^{q}, v\left|b_{i}\right|^{-p}\right), \quad i=1,2, \ldots, m,
$$

where $a_{i}, b_{i}$ are the functions in (1.3). Then the Hardy-type inequality (1.1) with kernel $k(x, t)$ from (1.3) holds if the weight functions $u, v$ satisfy for $i=1,2, \ldots, m$ the conditions

$$
\sup _{x>0} A_{M, i}(x)<\infty
$$

Proof Conditions (2.4) guarantee the validity of the Hardy inequality

$$
\left(\int_{0}^{\infty}\left|\int_{0}^{x} g(t) \mathrm{d} t\right|^{q} u(x)\left|a_{i}(x)\right|^{q} \mathrm{~d} x\right)^{\frac{1}{q}} \leq C_{1, i}\left(\int_{0}^{\infty}|g(t)|^{p} v(t)\left|b_{i}(t)\right|^{-p} \mathrm{~d} t\right)^{\frac{1}{p}}
$$

for functions $g$; if we take $g(t)=f(t) b_{i}(t)$, we can rewrite the foregoing Hardy inequality as

$$
\left(\int_{0}^{\infty}\left|\int_{0}^{x} a_{i}(x) b_{i}(t) f(t) \mathrm{d} t\right|^{q} u(x) \mathrm{d} x\right)^{\frac{1}{q}} \leq C_{1, i}\left(\int_{0}^{\infty}|f(t)|^{p} v(t) \mathrm{d} t\right)^{\frac{1}{p}}
$$

Now, using (1.3), the Minkowski and the last inequality, we obtain

$$
\begin{aligned}
\left(\int_{0}^{\infty}\left|\int_{0}^{x} k(x, t) f(t) \mathrm{d} t\right|^{q} u(x) \mathrm{d} x\right)^{\frac{1}{q}} & =\left(\int_{0}^{\infty}\left|\sum_{i=1}^{m} \int_{0}^{x} a_{i}(x) b_{i}(t) f(t) \mathrm{d} t\right|^{q} u(x) \mathrm{d} x\right)^{\frac{1}{q}} \\
& \leq \sum_{i=1}^{m}\left(\int_{0}^{\infty}\left|\int_{0}^{x} a_{i}(x) b_{i}(t) f(t) \mathrm{d} t\right|^{q} u(x) \mathrm{d} x\right)^{\frac{1}{q}} \\
& \leq \sum_{i=1}^{m} C_{1, i}\left(\int_{0}^{\infty}|f(t)|^{p} v(t) \mathrm{d} t\right)^{\frac{1}{p}}
\end{aligned}
$$

i.e., we have derived inequality (1.1).

Remark 2.3 Let us mention that the expression $A_{M, i}(x)$ in (2.3) is nothing else than the expression $A_{M}(x)$ from (1.9), where we replace $A, B$ by $A_{i}, B_{i}$, respectively, with $A_{i}, B_{i}$ from (1.5).

\section{Necessary conditions}

First let us introduce some auxiliary notions.

Definition 3.1 We will say that the system (matrix) $\left\{D_{i, j}\right\}_{i, j=1}^{m}$ of real numbers $D_{i, j}$ satisfies the ellipticity condition if there exists a constant $C_{E}>0$ such that

$$
\sum_{i, j=1}^{m} D_{i, j} \xi_{i} \xi_{j} \geq C_{E} \sum_{i=1}^{m} D_{i, i} \xi_{i}^{2} \quad \text { for all } \xi=\left\{\xi_{i}\right\}_{i=1}^{m} \in \mathbb{R}^{m}
$$


Remark 3.2 (i) The ellipticity condition is equivalent to the positive definiteness of the quadratic form

$$
\sum_{i, j=1}^{m} \tilde{D}_{i, j} \xi_{i} \xi_{j} \quad \text { with } \xi=\left\{\xi_{i}\right\}_{i=1}^{m} \in \mathbb{R}^{m},
$$

where

$$
\tilde{D}_{i, j}= \begin{cases}(1-c) D_{i, i}, & i=j, \\ D_{i, j}, & i \neq j\end{cases}
$$

with some $0<c<1$.

(ii) A sufficient condition for the ellipticity to be satisfied is

$$
D_{i, j} \leq(1-c) \sqrt{D_{i, i} D_{j, j}} \text { for } i \neq j \text {. }
$$

Indeed, if we denote $M^{-}=\left\{(i, j): D_{i, j} \xi_{i} \xi_{j}<0,1 \leq i, j \leq m\right\}$, then

$$
\begin{aligned}
\sum_{i, j=1}^{m} D_{i, j} \xi_{i} \xi_{j} & \geq \sum_{i=1}^{m} D_{i, i} \xi_{i}^{2}-\sum_{(i, j) \in M^{-}}\left|D_{i, j}\right|\left|\xi_{i}\right|\left|\xi_{j}\right| \\
& \geq \sum_{i=1}^{m} D_{i, i} \xi_{i}^{2}-\sum_{(i, j) \in M^{-}}(1-c) \sqrt{D_{i, i} D_{j, j}}\left|\xi_{i}\right|\left|\xi_{j}\right| \\
& \geq \sum_{i=1}^{m} D_{i, i} \xi_{i}^{2}-\sum_{(i, j) \in M^{-}} \frac{(1-c)}{2}\left(D_{i, i} \xi_{i}^{2}+D_{j, j} \xi_{j}^{2}\right) \\
& \geq c \sum_{i=1}^{m} D_{i, i} \xi_{i}^{2} .
\end{aligned}
$$

To find necessary conditions for (1.1) to hold, we consider three cases.

(I) The case $p=2,1<q<\infty$. Denote for this case

$$
A_{2}:=\sup _{z>0}\left(\int_{z}^{\infty}\left(\int_{0}^{z} k^{2}(x, t) v^{-1}(t) \mathrm{d} t\right)^{\frac{q}{2}} u(x) \mathrm{d} x\right)^{\frac{1}{q}} .
$$

Theorem 3.3 Let $p=2$ and $1<q<\infty$. Then the following condition

$$
A_{2}<\infty
$$

is necessary for inequality (1.1) to hold.

Proof For $z>0$ and for $\left\{B_{i}\right\}_{i=1}^{m}$ from (1.5) let $\left\{B_{i_{k}}\right\}_{k=1}^{n}$ be an arbitrary maximal linearly independent subsystem of $\left\{B_{i}\right\}_{i=1}^{m}$ in $L^{2}(0, z)$, which, for simplicity, we denote by $\left\{B_{i}\right\}_{i=1}^{n}$. Using the Gram-Schmidt method of orthogonalization to the system $\left\{B_{i}\right\}_{i=1}^{n}$ in $L^{2}(0, z)$, we obtain a system $\left\{B_{i, z}\right\}_{i=1}^{n}$ such that

$$
B_{i}(t)=\sum_{j=1}^{n} \beta_{i, j}(z) B_{j, z}(t)
$$


for $t \in(0, z)$ and $i=1,2, \ldots, m$. Using this we rewrite the kernel in (1.6) in the form

$$
\begin{aligned}
K(x, t) & =\sum_{i=1}^{m} A_{i}(x) B_{i}(t) \\
& =\sum_{i=1}^{m} A_{i}(x) \sum_{j=1}^{n} \beta_{i, j}(z) B_{j, z}(t) \\
& =\sum_{j=1}^{n} B_{j, z}(t) \sum_{i=1}^{m} \beta_{i, j}(z) A_{i}(x) \\
& =\sum_{j=1}^{n} A_{j, z}(x) B_{j, z}(t)=K_{z}(x, t),
\end{aligned}
$$

for $t \in(0, z)$, where $A_{j, z}(x)=\sum_{i=1}^{m} \beta_{i, j}(z) A_{i}(x)$.

Let $1 \leq j \leq n$, then choosing $f_{j, z}(t)=\chi_{(0, z)}(t) B_{j, z}(t)\left\|B_{j, z}\right\|_{L^{2}(0, z)}^{-1}$ and using the orthogonality of the system $\left\{B_{i, z}\right\}_{i=1}^{n}$, we estimate the left-hand side of (1.6) as

$$
\begin{aligned}
\left\|K f_{j, z}\right\|_{L^{q}} & =\left(\int_{0}^{\infty}\left|\int_{0}^{x} K(x, t) f_{j, z}(t) \mathrm{d} t\right|^{q} \mathrm{~d} x\right)^{\frac{1}{q}} \\
& \geq\left(\int_{z}^{\infty}\left|\int_{0}^{z} K(x, t) f_{j, z}(t) \mathrm{d} t\right|^{q} \mathrm{~d} x\right)^{\frac{1}{q}} \\
& =\left(\int_{z}^{\infty}\left|\int_{0}^{z} K_{z}(x, t) B_{j, z}(t) \mathrm{d} t\right|^{q} \mathrm{~d} x\right)^{\frac{1}{q}}\left\|B_{j, z}\right\|_{L^{2}(0, z)}^{-1} \\
& =\left(\int_{z}^{\infty}\left|\sum_{i=1}^{n} A_{i, z}(x) \int_{0}^{z} B_{i, z}(t) B_{j, z}(t) \mathrm{d} t\right|^{q} \mathrm{~d} x\right)^{\frac{1}{q}}\left\|B_{j, z}\right\|_{L^{2}(0, z)}^{-1} \\
& =\left(\int_{z}^{\infty}\left|A_{j, z}(x)\right|^{q} \mathrm{~d} x\right)^{\frac{1}{q}}\left(\int_{0}^{z}\left|B_{j, z}(t)\right|^{2} \mathrm{~d} t\right)^{\frac{1}{2}} .
\end{aligned}
$$

This estimate together with (1.6) implies

$$
\begin{aligned}
n C=C \sum_{j=1}^{n}\left\|f_{j, z}\right\|_{L^{2}} & \geq \sum_{j=1}^{n}\left\|K f_{j, z}\right\|_{L^{q}} \\
& \geq \sum_{j=1}^{n}\left(\int_{z}^{\infty}\left|A_{j, z}(x)\right|^{q} \mathrm{~d} x\right)^{\frac{1}{q}}\left(\int_{0}^{z}\left|B_{j, z}(t)\right|^{2} \mathrm{~d} t\right)^{\frac{1}{2}} \\
& :=A_{2}^{*}(z) .
\end{aligned}
$$

Using the Minkowski inequality, we estimate $A_{2}^{*}(z)$ in the form

$$
\begin{aligned}
A_{2}^{*}(z) & =\sum_{j=1}^{n}\left(\int_{z}^{\infty}\left[\left(\int_{0}^{z}\left|A_{j, z}(x) B_{j, z}(t)\right|^{2} \mathrm{~d} t\right)^{\frac{1}{2}}\right]^{q} \mathrm{~d} x\right)^{\frac{1}{q}} \\
& \geq\left(\int_{z}^{\infty}\left[\sum_{j=1}^{n}\left(\int_{0}^{z}\left|A_{j, z}(x) B_{j, z}(t)\right|^{2} \mathrm{~d} t\right)^{\frac{1}{2}}\right]^{q} \mathrm{~d} x\right)^{\frac{1}{q}}
\end{aligned}
$$




$$
\begin{aligned}
& \geq\left(\int_{z}^{\infty}\left(\int_{0}^{z}\left|\sum_{j=1}^{n} A_{j, z}(x) B_{j, z}(t)\right|^{2} \mathrm{~d} t\right)^{\frac{q}{2}} \mathrm{~d} x\right)^{\frac{1}{q}} \\
& =\left(\int_{z}^{\infty}\left(\int_{0}^{z}\left|\sum_{j=1}^{n} \sum_{i=1}^{m} A_{i}(x) \beta_{i, j}(z) B_{j, z}(t)\right|^{2} \mathrm{~d} t\right)^{\frac{q}{2}} \mathrm{~d} x\right)^{\frac{1}{q}} \\
& =\left(\int_{z}^{\infty}\left(\int_{0}^{z}\left|\sum_{i=1}^{m} \sum_{j=1}^{n} A_{i}(x) \beta_{i, j}(z) B_{j, z}(t)\right|^{2} \mathrm{~d} t\right)^{\frac{q}{2}} \mathrm{~d} x\right)^{\frac{1}{q}} \\
& =\left(\int_{z}^{\infty}\left(\int_{0}^{z}\left|\sum_{i=1}^{m} A_{i}(x) B_{i}(t)\right|^{2} \mathrm{~d} t\right)^{\frac{q}{2}} \mathrm{~d} x\right)^{\frac{1}{q}} \\
& =\left(\int_{z}^{\infty}\left(\int_{0}^{z} k^{2}(x, t) v^{-1}(t) \mathrm{d} t\right)^{\frac{q}{2}} u(x) \mathrm{d} x\right)^{\frac{1}{q}} .
\end{aligned}
$$

Since $z>0$ is arbitrary and inequality (1.1) (i.e., inequality (1.6)) holds, we conclude that $C$ is finite and we get (3.1).

Moreover, we can show that condition (2.4) is also necessary if we add some assumptions. For this purpose, denote

$$
D_{i, j}(z)=\int_{0}^{z} B_{i}(t) B_{j}(t) \mathrm{d} t=\int_{0}^{z} b_{i}(t) b_{j}(t) v^{-1}(t) \mathrm{d} t
$$

where $B_{i}(t)=b_{i}(t) v^{-1 / 2}(t)$.

Definition 3.4 We say that the condition $E_{2}$ is satisfied if there exists a constant $C_{E}$ such that for every $z>0$ the system $\left\{D_{i, j}(z)\right\}_{i, j=1}^{m}$ satisfies the ellipticity condition.

Theorem 3.5 Let $p=2$ and $1<q<\infty$. Suppose that the system $\left\{D_{i, j}(z)\right\}_{i, j=1}^{m}$ from (3.5) satisfies the condition $E_{2}$. Then (sufficient) condition (2.4) is necessary for (1.1) to hold.

Proof Using Theorem 3.3, formula (3.4), the condition $E_{2}$ and the Minkowski inequality, we obtain

$$
\begin{aligned}
\sum_{j=1}^{n}\left\|K f_{j, z}\right\|_{L^{q}} & \geq\left(\int_{z}^{\infty}\left(\int_{0}^{z} k^{2}(x, t) v^{-1}(t) \mathrm{d} t\right)^{\frac{q}{2}} u(x) \mathrm{d} x\right)^{\frac{1}{q}} \\
& =\left(\int_{z}^{\infty}\left(\int_{0}^{z} \sum_{i, j=1}^{m} a_{i}(x) a_{j}(x) b_{i}(t) b_{j}(t) v^{-1}(t) \mathrm{d} t\right)^{\frac{q}{2}} u(x) \mathrm{d} x\right)^{\frac{1}{q}} \\
& =\left(\int_{z}^{\infty}\left(\sum_{i, j=1}^{m} D_{i, j}(z) A_{i}(x) A_{j}(x)\right)^{\frac{q}{2}} \mathrm{~d} x\right)^{\frac{1}{q}} \\
& \geq \sqrt{C_{E}}\left(\int_{z}^{\infty}\left(\sum_{i=1}^{m} D_{i, i}(z) A_{i}(x)^{2}\right)^{\frac{q}{2}} \mathrm{~d} x\right)^{\frac{1}{q}} \\
& =\sqrt{C_{E}}\left(\int_{z}^{\infty}\left(\sum_{i=1}^{m} A_{i}(x)^{2} \int_{0}^{z} B_{i}(t)^{2} \mathrm{~d} t\right)^{\frac{q}{2}} \mathrm{~d} x\right)^{\frac{1}{q}}
\end{aligned}
$$




$$
\begin{aligned}
& \geq \sqrt{C_{E}}\left(\int_{z}^{\infty}\left|A_{i}(x)\right|^{q} \mathrm{~d} x\right)^{\frac{1}{q}}\left(\int_{0}^{z} B_{i}(t)^{2} \mathrm{~d} t\right)^{\frac{1}{2}} \\
& =\sqrt{C_{E}}\left(\int_{z}^{\infty}\left|a_{i}(x)\right|^{q} u(x) \mathrm{d} x\right)^{\frac{1}{q}}\left(\int_{0}^{z} b_{i}^{2}(t) v^{-1}(t) \mathrm{d} t\right)^{\frac{1}{2}} \\
& =\sqrt{C_{E}} A_{M, i}(z)
\end{aligned}
$$

for arbitrary $i: 1 \leq i \leq m$. Consequently, from (3.3) and (3.6), we get (2.4). The theorem is proved.

(II) The case $1<p<2,1<q<\infty$. Let us denote

$$
D_{i, l}^{j}(z)=\int_{0}^{z} \tilde{B}_{i}^{j}(t) \tilde{B}_{l}^{j}(t) \mathrm{d} t, \quad 1 \leq i, j, l \leq m,
$$

where $\tilde{B}_{i}^{j}(t):=B_{i}(t)\left|B_{j}(t)\right|^{\frac{p^{\prime}-2}{2}}$ belongs to $L^{2}(0, z)$ since

$$
\int_{0}^{z}\left|\tilde{B}_{i}^{j}(t)\right|^{2} \mathrm{~d} t \leq\left(\int_{0}^{z}\left|B_{i}(t)\right|^{p^{\prime}} \mathrm{d} t\right)^{\frac{2}{p^{\prime}}}\left(\int_{0}^{z}\left|B_{j}(t)\right|^{p^{\prime}} \mathrm{d} t\right)^{\frac{p^{\prime}-2}{2 p^{\prime}}}<\infty .
$$

Definition 3.6 We will say that the condition $E_{p}$ is satisfied if there exists a constant $C_{E}>0$ such that for every $z>0$ the system $\left\{D_{i, l}^{j}(z)\right\}_{i, l=1}^{m}$ satisfies the ellipticity condition for $j=1,2, \ldots, m$.

Theorem 3.7 Let $1<p<2$ and $1<q<\infty$. If the condition $E_{p}$ holds, then (2.4) is necessary for inequality (1.1) to hold.

Proof Let $z>0$ and let $1 \leq j \leq m$. Let $\left\{\tilde{B}_{i_{k}}^{j}\right\}_{k=1}^{n}$ be a maximal linearly independent subsystem of $\left\{\tilde{B}_{i}^{j}\right\}_{i=1}^{m}$ in $L^{2}(0, z)$, which, for simplicity, we denote by $\left\{\tilde{B}_{i}^{j}\right\}_{i=1}^{n}$. Thus, using the method of orthogonalization, we get an orthogonal system $\left\{\tilde{B}_{i, z}^{j}\right\}_{i=1}^{n}$ in $L^{2}(0, z)$ such that

$$
\tilde{B}_{i}^{j}(t)=\sum_{l=1}^{n} \beta_{i, l}^{j}(z) \tilde{B}_{l, z}^{j}(t), \quad i=1,2, \ldots, m .
$$

If we denote $g(t)=\tilde{B}_{l, z}^{j}(t)\left\|\tilde{B}_{l, z}^{j}\right\|_{L^{2}(0, z)}^{-1}, c_{j}=\left\|B_{j}\right\|_{L^{p^{\prime}}(0, z)}^{\frac{p-2}{2 p-2}}$ and choose the following test function in (1.6) as

$$
f_{l, z}^{j}(t)=\chi_{(0, z)}(t) c_{j}\left|B_{j}(t)\right|^{\frac{p^{\prime}-2}{2}} g(t)
$$

then the left- and right-hand sides are estimated in the forms:

$$
\begin{aligned}
\left\|f_{l, z}^{j}\right\|_{L^{p}} & =c_{j}\left(\int_{0}^{z}\left|B_{j}(t)\right|^{\frac{\left(p^{\prime}-2\right) p}{2}}|g(t)|^{p} \mathrm{~d} t\right)^{\frac{1}{p}} \\
& \leq c_{j}\left(\int_{0}^{z}\left|B_{j}(t)\right|^{p^{\prime}} \mathrm{d} t\right)^{\frac{2-p}{2 p}}\left(\int_{0}^{z}|g(t)|^{2} \mathrm{~d} t\right)^{\frac{1}{2}} \\
& =1,
\end{aligned}
$$


and

$$
\begin{aligned}
\left\|K f_{l, z}^{j}\right\|_{L^{q}} & =\left(\int_{0}^{\infty}\left|\int_{0}^{x} K(x, t) f_{l, z}^{j}(t) \mathrm{d} t\right|^{q} \mathrm{~d} x\right)^{\frac{1}{q}} \\
& \geq\left(\int_{z}^{\infty}\left|\int_{0}^{z} K(x, t) f_{l, z}^{j}(t) \mathrm{d} t\right|^{q} \mathrm{~d} x\right)^{\frac{1}{q}} \\
& =c_{j}\left(\int_{z}^{\infty}\left|\int_{0}^{z} \tilde{K}^{j}(x, t) g(t) \mathrm{d} t\right|^{q} \mathrm{~d} x\right)^{\frac{1}{q}},
\end{aligned}
$$

where $\tilde{K}^{j}(x, t)=\sum_{i=1}^{m} A_{i}(x) \tilde{B}_{i}^{j}(t)$.

Using (3.7) we rewrite the kernel $\tilde{K}^{j}(x, t)$ in the form

$$
\begin{aligned}
\tilde{K}^{j}(x, t) & =\sum_{i=1}^{m} A_{i}(x)\left[\sum_{l=1}^{n} \beta_{i, l}^{j}(z) \tilde{B}_{l, z}^{j}(t)\right] \\
& =\sum_{l=1}^{n} A_{l, z}^{j}(x) \tilde{B}_{l, z}^{j}(t)=\tilde{K}_{z}^{j}(x, t),
\end{aligned}
$$

where $A_{l, z}^{j}(x)=\sum_{i=1}^{m} A_{i}(x) \beta_{i, l}^{j}(z)$.

Then we have from (3.8), (3.9) and (1.6) that

$$
c_{j}\left(\int_{z}^{\infty}\left|\int_{0}^{z} \tilde{K}_{z}^{j}(x, t) g(t) \mathrm{d} t\right|^{q} \mathrm{~d} x\right)^{\frac{1}{q}} \leq\left\|K f_{l, z}^{j}\right\|_{L^{q}} \leq C .
$$

Now, repeating the proofs of the foregoing theorems with respect to (3.10) with the kernel $\tilde{K}_{z}^{j}(x, t)$, we can also get a similar estimate as in (3.2) in the form

$$
n C \geq \sum_{l=1}^{n}\left\|K f_{l, z}^{j}\right\|_{L^{q}} \geq c_{j} \sum_{l=1}^{n}\left(\int_{z}^{\infty}\left|A_{l, z}^{j}(x)\right|^{q} \mathrm{~d} x\right)^{\frac{1}{q}}\left(\int_{0}^{z}\left|\tilde{B}_{l, z}^{j}(t)\right|^{2} \mathrm{~d} t\right)^{\frac{1}{2}} .
$$

Then supposing the condition $E_{p}$, we also obtain the following estimate:

$$
\begin{aligned}
\sum_{l=1}^{n}\left\|K f_{l, z}^{j}\right\|_{L^{q}} & \geq c_{j} \sum_{l=1}^{n}\left(\int_{z}^{\infty}\left|A_{l, z}^{j}(x)\right|^{q} \mathrm{~d} x\right)^{\frac{1}{q}}\left(\int_{0}^{z}\left|B_{l, z}^{j}(t)\right|^{2} \mathrm{~d} t\right)^{\frac{1}{2}} \\
& \vdots \\
& \geq c_{j}\left(\int_{z}^{\infty}\left(\sum_{i, l=1}^{m} D_{i, l}^{j}(z) A_{i}(x) A_{l}(x)\right)^{\frac{q}{2}} \mathrm{~d} x\right)^{\frac{1}{q}} \\
& \geq c_{j} \sqrt{C_{E}}\left(\int_{z}^{\infty}\left(\sum_{i=1}^{m} D_{i, i}^{j}(z) A_{i}(x)^{2}\right)^{\frac{q}{2}} \mathrm{~d} x\right)^{\frac{1}{q}} \\
& \geq c_{j} \sqrt{C_{E}}\left(\int_{z}^{\infty}\left|A_{j}(x)\right|^{q} \mathrm{~d} x\right)^{\frac{1}{q}}\left(\int_{0}^{z}\left|B_{j}(t)\right|^{p^{\prime}} \mathrm{d} t\right)^{\frac{1}{2}},
\end{aligned}
$$


i.e.,

$$
\begin{aligned}
\sum_{l=1}^{n}\left\|K f_{l, z}^{j}\right\|_{L^{q}} & \geq c_{j} \sqrt{C_{E}}\left(\int_{z}^{\infty}\left|A_{j}(x)\right|^{q} \mathrm{~d} x\right)^{\frac{1}{q}}\left(\int_{0}^{z}\left|B_{j}(t)\right|^{p^{\prime}} \mathrm{d} t\right)^{\frac{1}{2}} \\
& \geq \sqrt{C_{E}}\left(\int_{z}^{\infty}\left|a_{j}(x)\right|^{q} u(x) \mathrm{d} x\right)^{\frac{1}{q}}\left(\int_{0}^{z}\left|b_{j}(t)\right|^{p^{\prime}} v^{1-p^{\prime}}(t) \mathrm{d} t\right)^{\frac{1}{p^{\prime}}} \\
& =\sqrt{C_{E}} A_{M, j}(z),
\end{aligned}
$$

which holds for $1 \leq j \leq m$ and $z>0$.

Using this estimate and (3.11), we finally get that

$$
\frac{\sqrt{C_{E}}}{n} \sup _{z>0} A_{M, j}(z) \leq C
$$

The proof is complete.

(III) The case $1<p<\infty, 2 \leq q<\infty$. Another approach how to investigate inequality (1.1) is based on the following lemma (for details, see [7]).

Lemma 3.8 Let $1<p, q<\infty$. Then inequality (1.6) holds for all $f \in L^{p}$ if and only if the conjugate inequality

$$
\left(\int_{0}^{\infty}\left|K^{*} g(x)\right|^{p^{\prime}} \mathrm{d} x\right)^{\frac{1}{p^{\prime}}} \leq C\left(\int_{0}^{\infty}|g(x)|^{q^{\prime}} \mathrm{d} x\right)^{\frac{1}{q^{\prime}}}
$$

holds for all $g \in L^{q^{\prime}}$, where

$$
K^{*} g(x):=\int_{x}^{\infty} K(t, x) g(t) \mathrm{d} t
$$

and $K(t, x)=\sum_{i=1}^{m} A_{i}(t) B_{i}(x)$.

The lemma enables to replace the investigation of inequality (1.6) by the investigation of inequality (3.13).

Denote

$$
D_{i, l}^{j}(z)=\int_{z}^{\infty} \tilde{A}_{i}^{j}(t) \tilde{A}_{l}^{j}(t) \mathrm{d} t, \quad 1 \leq i, j, l \leq m,
$$

where $\tilde{A}_{l}^{j}(t):=A_{l}(t)\left|A_{j}(t)\right|^{\frac{q-2}{2}}$ belongs to $L^{2}(z, \infty)$ since

$$
\int_{z}^{\infty}\left|\tilde{A}_{i}^{j}(t)\right|^{2} \mathrm{~d} t \leq\left(\int_{z}^{\infty}\left|A_{i}(t)\right|^{q} \mathrm{~d} t\right)^{\frac{2}{q}}\left(\int_{z}^{\infty}\left|A_{j}(t)\right|^{q} \mathrm{~d} t\right)^{\frac{q-2}{2 q}}<\infty .
$$

Definition 3.9 We will say that the condition $E_{q^{\prime}}$ is satisfied if there exists a constant $C_{E}>0$ and for every $z>0$ such that the system $\left\{D_{i, l}^{j}(z)\right\}_{i, l=1}^{m}$ satisfies the ellipticity condition for $j=1,2, \ldots, m$. 
Theorem 3.10 Let $1<p<\infty$ and $2 \leq q<\infty$. If the condition $E_{q^{\prime}}$ holds for the system from (3.15), then (2.4) is necessary for inequality (1.6) to hold.

Proof Let $z>0$ and let $1 \leq j \leq m$. Let $\left\{\tilde{A}_{i_{k}}^{j}\right\}_{k=1}^{n}$ be a maximal linearly independent subsystem of $\left\{\tilde{A}_{i}^{j}\right\}_{i=1}^{m}$ in $L^{2}(z, \infty)$, which, for simplicity, we denote by $\left\{\tilde{A}_{i}^{j}\right\}_{i=1}^{n}$. Thus, using the method of orthogonalization, we get an orthogonal system $\left\{\tilde{A}_{i, z}^{j}\right\}_{i=1}^{n}$ in $L^{2}(z, \infty)$ such that

$$
\tilde{A}_{i}^{j}(t)=\sum_{l=1}^{n} \alpha_{i, l}^{j}(z) \tilde{A}_{l, z}^{j}(t), \quad i=1,2, \ldots, m
$$

If we denote $g(t)=\tilde{A}_{l, z}^{j}(t)\left\|\tilde{A}_{l, z}^{j}\right\|_{L^{2}(z, \infty)}^{-1}, c_{j}=\left\|A_{j}\right\|_{L^{q}(z, \infty)}^{\frac{q^{\prime}-2}{2 q^{\prime}-2}}$ and choose the test function in the form

$$
f_{l, z}^{j}(t)=\chi_{(z, \infty)}(t) c_{j}\left|A_{j}(t)\right|^{\frac{q-2}{2}} g(t)
$$

then the left- and right-hand sides are similarly estimated in the forms:

$$
\left\|f_{l, z}^{j}\right\|_{L^{q^{\prime}}} \leq 1
$$

and

$$
\left\|K^{*} f_{l, z}^{j}\right\|_{L^{p^{\prime}}} \geq c_{j}\left(\int_{0}^{z}\left|\int_{z}^{\infty} \tilde{K}^{j}(t, x) g(t) \mathrm{d} t\right|^{p^{\prime}} \mathrm{d} x\right)^{\frac{1}{p^{\prime}}}
$$

where $\tilde{K}^{j}(t, x)=\sum_{i=1}^{m} \tilde{A}_{i}^{j}(t) B_{i}(x)$

Using (3.7) we can rewrite the kernel $\tilde{K}^{j}(t, x)$ in the form

$$
\begin{aligned}
\tilde{K}^{j}(t, x) & =\sum_{i=1}^{m} B_{i}(x)\left[\sum_{l=1}^{n} \alpha_{i, l}^{j}(z) \tilde{A}_{l, z}^{j}(t)\right] \\
& =\sum_{l=1}^{n} \tilde{A}_{l, z}^{j}(t) B_{l, z}^{j}(x)=\tilde{K}_{z}^{j}(t, x),
\end{aligned}
$$

where $B_{l, z}^{j}(x)=\sum_{i=1}^{m} B_{i}(x) \alpha_{i, l}^{j}(z)$.

Then we have from (3.17), (3.18) and (3.13) that

$$
c_{j}\left(\int_{0}^{z}\left|\int_{z}^{\infty} \tilde{K}_{z}^{j}(t, x) g(t) \mathrm{d} t\right|^{p^{\prime}} \mathrm{d} x\right)^{\frac{1}{p^{\prime}}} \leq C .
$$

Now repeating the proof of the foregoing theorem with respect to (3.19) with the kernel $\tilde{K}_{z}^{j}(t, x)$, we can also get a similar estimate as in (3.11) in the form

$$
n C \geq \sum_{l=1}^{n}\left\|K^{*} f_{l, z}^{j}\right\|_{L^{p^{\prime}}} \geq c_{j} \sum_{l=1}^{n}\left(\int_{0}^{z}\left|B_{l, z}^{j}(t)\right|^{p^{\prime}} \mathrm{d} t\right)^{\frac{1}{p^{\prime}}}\left(\int_{z}^{\infty}\left|\tilde{A}_{l, z}^{j}(x)\right|^{2} \mathrm{~d} x\right)^{\frac{1}{2}} .
$$


Then, supposing that the condition $E_{q^{\prime}}$ is satisfied, we also obtain the following estimate:

$$
\begin{aligned}
\sum_{l=1}^{n}\left\|K^{*} f_{l, z}^{j}\right\|_{L^{p^{\prime}}} & \geq c_{j} \sum_{l=1}^{n}\left(\int_{0}^{z}\left|B_{l, z}^{j}(t)\right|^{p^{\prime}} \mathrm{d} t\right)^{\frac{1}{p^{\prime}}}\left(\int_{z}^{\infty}\left|\tilde{A}_{l, z}^{j}(x)\right|^{2} \mathrm{~d} x\right)^{\frac{1}{2}} \\
& \vdots \\
& \geq c_{j}\left(\int_{z}^{\infty}\left(\sum_{i, l=1}^{m} D_{i, l}^{j}(z) B_{i}(x) B_{l}(x)\right)^{\frac{p^{\prime}}{2}} \mathrm{~d} x\right)^{\frac{1}{p^{\prime}}} \\
& \geq c_{j} \sqrt{C_{E}}\left(\int_{z}^{\infty}\left(\sum_{i=1}^{m} D_{i, i}^{j}(z)\left|B_{i}(x)\right|^{2}\right)^{\frac{p^{\prime}}{2}} \mathrm{~d} x\right)^{\frac{1}{p^{\prime}}} \\
& \geq c_{j} \sqrt{C_{E}}\left(\int_{z}^{\infty}\left|B_{j}(x)\right|^{p^{\prime}} \mathrm{d} x\right)^{\frac{1}{p^{\prime}}}\left(\int_{0}^{z}\left|A_{j}(t)\right|^{q} \mathrm{~d} t\right)^{\frac{1}{2}}
\end{aligned}
$$

i.e.,

$$
\begin{aligned}
\sum_{l=1}^{n}\left\|K^{*} f_{l, z}^{j}\right\|_{L^{p^{\prime}}} & \geq c_{j} \sqrt{C_{E}}\left(\int_{z}^{\infty}\left|A_{j}(x)\right|^{q} \mathrm{~d} x\right)^{\frac{1}{q}}\left(\int_{0}^{z}\left|B_{j}(t)\right|^{p^{\prime}} \mathrm{d} t\right)^{\frac{1}{2}} \\
& \geq \sqrt{C_{E}}\left(\int_{z}^{\infty}\left|a_{j}(x)\right|^{q} u(x) \mathrm{d} x\right)^{\frac{1}{q}}\left(\int_{0}^{z}\left|b_{j}(t)\right|^{p^{\prime}} v^{1-p^{\prime}}(t) \mathrm{d} t\right)^{\frac{1}{p^{\prime}}} \\
& =\sqrt{C_{E}} A_{M, j}(z)
\end{aligned}
$$

which holds for all $1 \leq j \leq m$ and $z>0$.

Using this estimate and (3.20), we finally get that

$$
\frac{\sqrt{C_{E}}}{n} \sup _{z>0} A_{M, j}(z) \leq C .
$$

The proof is complete.

\section{Criteria of compactness}

As far as the compactness of the imbedding $k: L^{p}(v) \rightarrow L^{q}(u)$ is concerned, we have the following.

Theorem 4.1 Let $1<p \leq q<\infty$. Let us suppose that the functions $A_{M, i}(x)$ from (2.4) satisfy

$$
\lim _{x \rightarrow 0+} A_{M, i}(x)=\lim _{x \rightarrow \infty} A_{M, i}(x)=0, \quad i=1,2, \ldots m
$$

Then the operator $k$ from (1.4) maps $L^{p}(v)$ into $L^{q}(u)$ compactly.

Proof Conditions (4.1) guarantee that the operators of $k_{i}$ from $L^{p}(v)$ into $L^{q}(u)$ are compact (see, e.g., [7]). Since $k=\sum_{i=1}^{m} k_{i}$, the compactness of $k$ follows.

Theorem 4.2 Let $1<p \leq 2$ and $1<q<\infty$. Let the condition $E_{p}$ be satisfied. If the operator $k$ from (1.4) is compact from $L^{p}(v)$ to $L^{q}(u)$, then conditions (2.4) and (4.1) are satisfied. 
Proof Let us suppose that operator (1.4) is compact. Then it is bounded, and, by using Theorems 3.3 and 3.7, we get (2.4) and also the compactness of the operator

$$
K f(x)=\int_{0}^{x} \sum_{i=1}^{m} A_{i}(x) B_{i}(t) f(t) \mathrm{d} t
$$

as an operator from $L^{p}$ to $L^{q}$.

Let $z>0$ and $1 \leq j, l \leq m$. Moreover, we choose the function $f_{l, z}^{j}(t)$ as in the proof of Theorem 3.7. Let $h \in L^{p^{\prime}}$ be arbitrary, then using (3.8) we have

$$
\begin{aligned}
\left|\int_{0}^{\infty} h(t) f_{l, z}^{j}(t) \mathrm{d} t\right| & \leq\left(\int_{0}^{z}|h(t)|^{p^{\prime}} \mathrm{d} t\right)^{\frac{1}{p^{\prime}}}\left(\int_{0}^{z}\left|f_{l, z}^{j}(t)\right|^{p} \mathrm{~d} t\right)^{\frac{1}{p}} \\
& \leq\left(\int_{0}^{z}|h(t)|^{p^{\prime}} \mathrm{d} t\right)^{\frac{1}{p^{\prime}}}
\end{aligned}
$$

from which it follows that

$$
\int_{0}^{\infty} h(t) f_{j, z}(t) \mathrm{d} t \rightarrow 0
$$

as $z \rightarrow 0+$, i.e., the class of functions $f_{l, z}^{j}$ weakly converges to zero in $L^{p}$ as $z \rightarrow 0+$.

This and the compactness of operator (4.2) imply that the class of images $K f_{l, z}^{j}$ strongly converges to zero in $L^{q}$ as $z \rightarrow 0+$, i.e.,

$$
\left\|K f_{l, z}^{j}\right\|_{L^{q}} \rightarrow 0 \quad \text { as } z \rightarrow 0+
$$

Analogously as in the proof of Theorem 3.7, (3.12) can also be obtained, i.e.,

$$
\sum_{l=1}^{n}\left\|K f_{l, z}^{j}\right\|_{L^{q}} \geq \sqrt{C_{E}} A_{M, j}(z),
$$

which with (4.3) implies that $\lim _{z \rightarrow 0+} A_{M, j}(z)=0$ for all $1 \leq j \leq m$.

Now we show that $\lim _{z \rightarrow \infty} A_{M, j}(z)=0$ for all $1 \leq j \leq m$. The compactness of operator (4.2) follows from the compactness of the conjugate operator $K^{*}$ (3.14) from $L^{q^{\prime}}$ to $L^{p^{\prime}}$.

Let $h \in L^{q^{\prime}}$ and choose $h_{z}(t)=\chi_{(z, \infty)}(t) h(t) /\|h\|_{L^{q^{\prime}}(z, \infty)}$. It can be shown as in foregoing cases that the class of functions $\left\{h_{z}, z \in(0, \infty)\right\}$ weakly converges to zero in $L^{q^{\prime}}$ as $z \rightarrow \infty$. Then the class of images $K^{*} h_{z}$ strongly converges to zero in $L^{p^{\prime}}$ as $z \rightarrow \infty$, i.e.,

$$
\left\|K^{*} h_{z}\right\|_{L^{p^{\prime}}} \rightarrow 0 \quad \text { as } z \rightarrow \infty
$$

Using the dual principle of $L^{p^{\prime}}$, we have

$$
\begin{aligned}
\left\|K^{*} h_{z}\right\|_{L^{p^{\prime}}} & =\sup _{\substack{f \in L^{p} \\
\|f\|_{L^{p}} \leq 1}} \int_{0}^{\infty} f(t) K^{*} h_{z}(t) \mathrm{d} t \\
& =\sup _{\substack{f \in L^{p} \\
\|f\|_{L^{p}} \leq 1}} \int_{0}^{\infty} h_{z}(x)\left(\int_{0}^{x} K(x, t) f(t) \mathrm{d} t\right) \mathrm{d} x .
\end{aligned}
$$


Now, choosing $f_{l, z}^{j}$ as in the proof of Theorem 3.7 instead of $f$ in (4.6), we have

$$
\begin{aligned}
\left\|K^{*} h_{z}\right\|_{L^{p^{\prime}}} & \geq \int_{z}^{\infty} h_{z}(x) \int_{0}^{x} \sum_{i=1}^{m} A_{i}(x) \tilde{B}_{i}^{j}(t) g(t) \mathrm{d} t \mathrm{~d} x \\
& =\int_{z}^{\infty} h_{z}(x) \int_{0}^{z} \sum_{i=1}^{m} A_{i, z}^{j}(x) \tilde{B}_{i, z}^{j}(t) g(t) \mathrm{d} t \mathrm{~d} x \\
& \geq c_{j} \int_{z}^{\infty} h_{z}(x) A_{l, z}^{j}(x) \mathrm{d} x \int_{0}^{z}\left|\tilde{B}_{l, z}^{j}(t)\right|^{2} \mathrm{~d} t\left\|\tilde{B}_{l, z}^{j}\right\|_{L^{2}(0, z)}^{-1} \\
& \geq c_{j} \int_{z}^{\infty} h_{z}(x) A_{l, z}^{j}(x) \mathrm{d} x\left(\int_{0}^{z}\left|\tilde{B}_{l, z}^{j}(t)\right|^{2} \mathrm{~d} t\right)^{\frac{1}{2}} .
\end{aligned}
$$

Since the operator $K$ is bounded from $L^{p}$ to $L^{q}$, i.e., (3.10) is satisfied, from which we will have that $A_{l, z}^{j} \in L^{q}(z, \infty), z>0$. Then, choosing the function $h_{l, z}^{j}=A_{l, z}^{j}\left|A_{l, z}^{j}\right|^{q-2} \chi_{(z, \infty)}$, $h_{l, z}^{j}(t):=h_{z}(t)$ and from (4.7), we have

$$
\left\|K^{*} h_{l, z}^{j}\right\|_{L^{p^{\prime}}} \geq c_{j}\left(\int_{z}^{\infty}\left|A_{l, z}^{j}(x)\right|^{q} \mathrm{~d} x\right)^{\frac{1}{q}}\left(\int_{0}^{z}\left|\tilde{B}_{l, z}^{j}(t)\right|^{2} \mathrm{~d} t\right)^{\frac{1}{2}} .
$$

As in the proof of Theorem 3.7, by estimating the right-hand side, we obtain also that

$$
\sum_{l=1}^{n}\left\|K^{*} h_{l, z}^{j}\right\|_{L^{p^{\prime}}} \geq \sqrt{C_{E}} A_{M, j}(z)
$$

Consequently, from this and (4.5) we have that $\lim _{z \rightarrow \infty} A_{M, j}(z)=0$ for all $1 \leq j \leq m$.

The theorem is proved.

Theorem 4.3 Let $1<p<\infty$ and $2 \leq q<\infty$. Let the condition $E_{q^{\prime}}$ be satisfied. If operator (1.4) is compact from $L^{p}(v)$ to $L^{q}(u)$, then conditions (2.4) and (4.1) are satisfied.

Proof First we show that $\lim _{z \rightarrow \infty} A_{M, j}(z)=0$, for which we use the compactness of the dual operator $K^{*}$ and the proof of Theorem 3.10. Then it can be shown that the class of functions $f_{l, z}^{j}$ also weakly converges to zero in $L^{q^{\prime}}$ as $z \rightarrow \infty$ and

$$
\left\|K^{*} f_{l, z}^{j}\right\|_{L^{q}} \rightarrow 0 \quad \text { as } z \rightarrow \infty
$$

Analogously as in the proof of Theorem 3.10, (3.21) can be obtained, i.e.,

$$
\sum_{l=1}^{n}\left\|K^{*} f_{l, z}^{j}\right\|_{L^{p^{\prime}}} \geq \sqrt{C_{E}} A_{M, j}(z)
$$

which with (4.8) implies that $\lim _{z \rightarrow \infty} A_{M, j}(z)=0$ for all $1 \leq j \leq m$.

To prove $\lim _{z \rightarrow 0+} A_{M, j}(z)=0$, we use the duality principle as in the proof of the foregoing theorem. The formulation of the corresponding proof is left to the reader as an exercise. 
Corollary 4.4 Let $1<p \leq 2$ and $p \leq q<\infty$. Let us suppose that the condition $E_{p}$ is satisfied. Then operator (1.4) from $L^{p}(v)$ into $L^{q}(u)$ is bounded and compact if and only if (2.4) and (4.1) are satisfied, respectively.

Corollary 4.5 Let $2 \leq q<\infty$ and $1<p \leq q$. Let us suppose that the condition $E_{q^{\prime}}$ is satisfied. Then operator (1.4) from $L^{p}(v)$ into $L^{q}(u)$ is bounded and compact if and only if (2.4) and (4.1) are satisfied, respectively.

Corollary 4.6 Let $1<p \leq q<\infty$. Let us suppose that the conditions $E_{p}$ and $E_{q^{\prime}}$ are satisfied. Then operator (1.4) from $L^{p}(v)$ into $L^{q}(u)$ is bounded and compact if and only if (2.4) and (4.1) are satisfied, respectively.

\section{Competing interests}

The authors declare that they have no competing interests.

\section{Authors' contributions}

All authors contributed in all parts in equal extent, and read and approved the final manuscript.

\section{Acknowledgements}

The first author was supported by RVO: 67985840. The second author was supported by Leverhulme Trust; grant No. RPG-167 and Wales Institute of Mathematical \& Computational Sciences. The third author was supported by the Scientific Committee of Ministry of Education and Science of the Republic of Kazakhstan, grant No.1529/GF, on priority area 'Intellectual potential of the country'.

Received: 17 January 2013 Accepted: 17 June 2013 Published: 3 July 2013

\section{References}

1. Stepanov, VD: Two-weight estimates for Riemann-Liouville integrals. Math. USSR, Izv. 36(3), 669-681 (1991)

2. Oinarov, R: Two-sided norm estimates for certain classes of integral operators. Proc. Steklov Inst. Math. 204(3), 205-214 (1994)

3. Oinarov, R: Boundedness and compactness of Volterra type integral operators. Sib. Math. J. 48(5), 884-896 (2007)

4. Oinarov, R: Boundedness of integral operators from weighted Sobolev space to weighted Lebesgue space. Complex Var. Elliptic Equ. 56(10-11), 1021-1038 (2011)

5. Kokilashvili, V, Meskhi, A, Persson, L-E: Weighted Norm Inequalities for Integral Transforms with Product Kernels. Nova Publ., New York (2010)

6. Kufner, A, Maligranda, L, Persson, L-E: The Hardy Inequality - About Its History and Some Related Results. Vydavatelský Servis, Plzeň (2007). ISBN:978-80-86843-15-5

7. Kufner, A, Persson, L-E: Weighted Inequalities of Hardy Type, xviii+357pp. World Scientific, River Edge (2003). ISBN:981-238-195-3

8. Rychkov, VS: Splitting of Volterra integral operators with degenerate kernels. Proc. Steklov Inst. Math. 3(214), 260-278 (1997)

9. Kufner, A, Kuliev, K, Persson, L-E: Some higher order Hardy inequalities. J. Inequal. Appl. 69(1), 1-14 (2012)

doi:10.1186/1029-242X-2013-310

Cite this article as: Kufner et al.: Some criteria for boundedness and compactness of the Hardy operator with some special kernels. Journal of Inequalities and Applications 2013 2013:310.

\section{Submit your manuscript to a SpringerOpen ${ }^{\ominus}$ journal and benefit from:}

- Convenient online submission

- Rigorous peer review

- Immediate publication on acceptance

- Open access: articles freely available online

- High visibility within the field

- Retaining the copyright to your article 